\title{
Capsule Commentary on Garland et al., Randomized Controlled Trial of Brief Mindfulness Training and Hypnotic Suggestion for Acute Pain Relief in the Hospital Setting
}

\author{
Emily S. Wang, MD, FACP \\ Division of General and Hospital Medicine, University of Texas Health San Antonio and South Texas Veterans Health Care System, San Antonio, TX, \\ USA.
}

J Gen Intern Med 32(10): 1133

DOI: $10.1007 / \mathrm{s} 11606-017-4130-y$

(c) Society of General Internal Medicine 2017

$\mathrm{T}$ he national opioid epidemic is causing significant health consequences, and increased medical use of opioid analgesics has contributed to the crisis. ${ }^{1}$ According to a study previously published in JGIM, opioid-naïve patients discharged from the hospital with opioid prescriptions were more likely to be future chronic opioid users. ${ }^{2}$ Therefore, in balancing the importance of adequate pain control while minimizing the risk of addiction, finding effective non-narcotic and nonpharmacologic interventions would be ideal.

In this issue, Garland et al. conducted a study in the inpatient setting on acute pain control using non-pharmacologic, psychosocial interventions. ${ }^{3}$ Consenting, eligible participants were randomized to receive one of three single, 15-min interventions delivered by a trained social worker: (1) mindfulness training, (2) self-hypnosis or, (3) psychoeducation session providing empathetic responses to the patient and discussion of perception of pain control by behavioral coping strategies. Mindfulness and self-hypnosis interventions were based on prior validated research. In this study a single session of mindfulness training or self-hypnotic suggestion reduced immediate acute pain intensity, with a third of patients experiencing at least a $30 \%$ reduction in acute pain.

Although the patients were randomized and adjustments were made for baseline differences, there are questions that should be kept in mind regarding the effectiveness of the interventions. When comparing the baseline demographics, patients who underwent self-hypnotic suggestion received a higher average dose of morphine in the $24 \mathrm{~h}$ before the intervention. Interestingly, in the analysis of secondary outcomes, these same participants had a lower desire for opioids than did participants in the mindfulness or psychoeducation groups. What was not specifically addressed in the study is that for patients receiving narcotics, the type, mode of delivery, and timing of these medications with the intervention may affect the outcome. In addition, pain was measured immediately before and after the intervention. Given that these techniques could be learned and used by participants, it would be interesting to see whether the intervention could have a more sustained benefit. Regardless, these results begin to explore the potential non-pharmacologic possibilities for pain control. As called for by the JGIM Editor's Desk, health care providers and team members should be part of the solution to prescription drug abuse. ${ }^{4}$ Exploring alternatives to opiates for the management of inpatient pain is an important research agenda.

Corresponding Author: Emily S. Wang, MD, FACP; Division of General and Hospital MedicineUniversity of Texas Health San Antonio and South Texas Veterans Health Care System, San Antonio, TX, USA (e-mail: wange@uthscsa.edu).

\section{Compliance with Ethical Standards:}

Conflict of Interest: The author has no conflicts of interest with this article.

\section{REFERENCES}

1. Atluri S, Sudarshan G, Manchikant L. Assessment of the trends in medical use and misuse of opioid analgesics from 2004 to 2011. Pain Physician. 2014. 17(2):E119-28.

2. Garland EL, Baker AK, Larson P, et al. Randomized Controlled Trial of Brief Mindfulness Training and Hypnotic Suggestion for Acute Pain Relief in the Hospital Setting. J Gen Intern Med. doi: 10.1007/s11606-0174116-9.

3. Calcaterra SL, Yamashita TE, Min SJ, Keniston A, Frank JW, Binswanger IA. Opioid Prescribing at Hospital Discharge Contributes to Chronic Opioid Use. J Gen Intern Med. 2016. 31(5):478-85.

4. Feldman MD. Chronic Pain and Prescription Drug Use and Abuse: Emerging Research in General Internal Medicine. J Gen Intern Med. 2016. 31(5):451-2. 